\title{
LA INTERCULTURALIDAD A TRAVÉS DE UN VOLUNTARIADO INTERNACIONAL EN LA PENÍNSULA DE SANTA ELENA
}

\section{INTERCULTURALITY THROUGH AN INTERNATIONAL VOLUNTEER IN THE PENINSULA OF SANTA ELENA}

\author{
Daniel Fernando Guevara Gavilanes. Tnlg. \\ fisico18@hotmail.com
}

\section{RESUMEN}

Partiendo de la riqueza histórica, cultural, paisajista y actividades económicas de la provincia de Santa Elena, el autor analiza la gran riqueza ancestral de la Península y las posibilidades para desarrollar un programa de voluntariado internacional denominado "Peninsula Volunteers", basado en su experiencia como ex voluntario del programa de intercambio "Canadá Word Youth" y ex participante del programa "Center for Cultural Interchange" en USA; está dirigido a estudiantes extranjeros que deseen aprender español, vivir la interculturalidad desde una comuna y tener una experiencia laboral en los paisajes de la fascinante Península.

Palabras clave: interculturalidad, voluntariado, experiencia laboral.

\section{ABSTRACT}

Based on the historical, cultural and landscaped and economic activities of the province of Santa Elena, the author analyzes the vast ancestral wealth of the peninsula and the possibilities for developing an international volunteer program called "Peninsula Volunteers", based on his experience as former volunteer exchange program "Youth Canada Word " and former participant of the " Center for Cultural interchange " program in the USA; It is aimed at foreign students who want to learn Spanish, live interculturality from a commune and have work experience in the fascinating landscapes of the Peninsula.

Keywords: multiculturalism, volunteer work experience.

Recibido: noviembre de 2015 Aprobado: febrero de 2016

\section{Introducción}

Cultivar la empatía, convivir, acceder a la compresión del prójimo no es fácil. Comprender requiere prioritariamente un proceso de autoexplicación de cada uno para luchar contra un enemigo interior, que siempre tiende a atribuir a los demás la culpa, ya sea por su etnia, por su religión, por su lugar de procedencia, por su género, su edad, etc.; La comprensión es un componente capital de la vida. Como dice Edgar Morin (2011:251)“No podemos esperar el menor progreso social sino va ligado a un progreso en la comprensión del prójimo”.

La mayoría de veces somos incapaces de considerar la complejidad de un ser humano, puesto que ni en las escuelas ni en la mayoría de los hogares, nos enseñan que el comprender, prioritariamente requiere un proceso de autoevaluación que revele la importancia de los prejuicios y paradigmas que inconscientemente gobiernan nuestras las de conocimiento y nos hacen incapaces de comprender que los demás también obedecen a otros preconceptos y otros paradigmas. ¿Por qué entonces, no encontrar una vía que permita no reducir a ese "otro" a su peor comportamiento dando lugar a malos entendidos, incomprensiones y hostilidades?

En esta perspectiva, se entiende que un viaje de intercambio cultural constituye un momento particular para comprender la interculturalidad desde sus diferentes ámbitos. Desde el aprendizaje para el conocimiento de uno mismo hasta una apertura respetuosa hacia la diversidad, que enriquece las relaciones mutuas con el aporte de los componentes socio-culturales de cada parte, sin que se diluyan nuestras identidades específicas.

En el año 1999, tuve la oportunidad de viajar a Canadá, a través del Programa de Intercambio "Canada 
World Youth", en el que conviví con indígenas nativos denominados "Cree" en la localidad de Moose Factory, ubicado en James Bay, Provincia de Ontario, Canadá.

En mi estadía de tres meses pude observar, cómo los nativos fueron desintegrados a través del proceso de colonización de ingleses y franceses. La sociedad Hydro-Quebec compró sus terrenos para construir una presa y un lago artificial. El lago cortó la ruta que alimentaban a los Crees, y los peces, infectados de mercurio, ya no eran comestibles. Los hombres fueron privados de su modo de vida tradicional. Las mujeres, instaladas en casa al estilo occidental, encontraron cocinas, refrigeradoras y todo tipo de comodidades pero, al cambiar de alimentación y modo de vida, se generó un problema de obesidad. El alcoholismo hizo estragos en muchos jóvenes y niños. En general, la droga, la prostitución, la delincuencia y la degradación se apoderaron de estas poblaciones.

Como argumenta Walsh (2009) la interculturalidad como proyecto nos convoca a transgredir el racismo, la racialización, deshumanización de unos, o sobre humanización de otros, y romper con la subalternización de seres, saberes y formas de vida; lo que implica varias dimensiones de transformación de estructuras. Por lo tanto, el planteamiento intercultural, atañe, no solo a relaciones interétnicas, entre personas o grupos de diversa identidad cultural, sino a un nivel mucho más amplio.

La interculturalidad va más allá de un concepto; es una forma de vida, donde se potencia el respeto a las diferencias pero en igualdad de condiciones. En este enfoque, no se considera una cultura subordinada a otra, sino que cada una debe reconocerse como válida y para ello debe propiciarse un diálogo franco basado en relaciones horizontales y respetuosas que favorezcan una convivencia pacífica.

Al hablar de interculturalidad, no hablamos del contacto exclusivamente folklórico con las poblaciones, sino con su verdadera convivencia en comunidad, en donde tenga lugar la reafirmación personal y la integración con los demás desde una relación de comprensión y respeto. La incomprensión de lo lejano y también de lo próximo es evidente en nuestra actual sociedad.

Para ello, requerimos de una forma de educación que permita afrontar la relación con los demás a través de la comprensión, la humanización de las emociones y el respeto democrático. La interculturalidad nos permite un aprendizaje que se va desarrollado en la medida en que se posibilita el diálogo cultural el encuentro permanente con otras personas, la visita constante a otros lugares y diferentes culturas. La interculturalidad es: aceptar y disfrutar de las diferentes formas de expresión y operatividad de los "otros", rompiendo viejos marcos de referencia uniformadores y generando nuevos conocimientos.

En este sentido, la propuesta de un Programa de Voluntariado en la Península de Santa Elena, pretende promover la convivencia de dos a más culturas, por un determinado tiempo en una de las comunidades ancestrales de la Península de Santa Elena, en donde, además de los atractivos que posee su territorio, sus pobladores mantienen sus rasgos característicos, su sabiduría ancestral, sus tradiciones, costumbres, en donde las dos culturas, sin perder su autonomía; se beneficien de una experiencia de vida en el encuentro.

Cuando se habla de interculturalidad, se debe incorporar también el concepto de ética, con el fin de garantizar que la convivencia entre personas de diferentes culturas, este basada en el reconocimiento de sus derechos. Estamos conscientes de los riesgos desintegradores que puede provocar una pretensión de integración sin el componente ético.

Los pueblos indígenas y afrodescendientes, estuvieron durante mucho tiempo excluidos del pluralismo político. Se trataba de un pluralismo que les era inherentemente hostil, no solo porque no los reconocía como ciudadanos, sino también porque se basaba en premisas filosóficas y culturales que contradecían sus prácticas colectivas, sus formas de vida y de organización social. El propio marxismo mantuvo intacta la exclusión e inclusive la invisibilidad social y política de los pueblos indígenas, salvo algunas brillantes excepciones. A pesar de todo esto, la izquierda latinoamericana, tanto la marxista como la no marxista, mantuvo, en general, una actitud racista y colonialista hacia los pueblos indígenas, que a veces se traducía en exclusión y persecución, y otras veces en tutela paternalista y captación clientelista. (Santos, Bonaventura, 2012:20)

Por otro lado, Walsh (2009: 15) articula el concepto de interculturalidad con el de de-colonialismo, utiliza el término de-colonial en el sentido de no simplemente "desarmar, deshacer o revertir lo colonial... pasar de un momento colonial a uno no colonial. La intención, más bien, es señalar y provocar un posicionamiento - una postura y actitud continua- de transgredir, intervenir, in-surgir e incidir" para la búsqueda de construcciones alternativas. En ese sentido, la interculturalidad es un recurso para promover la armonía en el desarrollo 
personal y social, que a la vez asegure la unidad, con respeto, reconocimiento y la valoración de la diversidad.

En este orden de ideas, la construcción de la interculturalidad requiere del fomento de relaciones interpersonales y grupales positivas: estimular actitudes de apertura, convivencia, conocimiento y reconocimiento mutuo. Hablar de interculturalidad implica estimular actitudes de apertura y convivencia entre los/as distintos/as (en términos étnicos, culturales, de género, de opción sexual, entre generaciones) respetando las formas simbólicas, expresivas y operativas de cada uno, y evitando imponer estilos uniformizadores.

La interculturalidad es visualizada como la adquisición de competencias o capacidades para valorar la diversidad, convivir y relacionarse entre sí de manera creativa y positiva, estimulando relaciones dialógicas, y propendiendo a un acercamiento entre diversos/as. En esta mirada, la interculturalidad se presenta como un proceso en construcción.

Adquirimos esas competencias de una manera dinámica a través de la incorporación de nuevos referentes que generalmente los hallamos cuando conocemos nuevas personas y lugares. Un Programa de voluntariado, en ese sentido, resulta propicio para vivir y promover la interculturalidad, puesto que trae enormes beneficios tanto para el participante como para la comunidad que lo acoge, al promover el diálogo entre saberes entre diferentes culturas de manera equitativa.

\section{Potencialidades turísticas de la Pe- nínsula de Santa Elena}

Sobre la Península se han escrito varios libros que permiten conocer su historia, tradiciones, comida típica, folklore, etc., pero no todo está documentado, así que conocer a los pobladores autóctonos descendientes de los Guancavilcas y experimentar la convivencia a través de un Programa de Voluntariado, resulta una eficaz forma de conocer y experimentar la interculturalidad.

El programa se desarrollaría en comunas ancestrales, tales como: Sinchal, Barcelona, Cadeate, Chanduy, Valdivia, entre otras (Anexo1)

Para ello, será necesario elaborar una base de datos de familias anfitrionas en los tres cantones de la Península, así como de los establecimientos y empresas que estén dispuestas a brindar las facilidades necesarias para que los participantes realicen su trabajo de voluntariado. Así también se podría establecer un convenio de cooperación interinstitucional con la Universidad Península de Santa Elena- UPSE, para que los participantes del voluntariado puedan realizar pasantías en proyectos que mantengan la Facultad de Ciencias Agrarias, la escuela de Turismo y la de Biología Marina.

La Península de Santa Elena está ubicada en el punto más extremo de la costa ecuatoriana, posee una extensión de 3.762,8 Km2, está conformada por tres cantones: el cantón Salinas con una extensión de 68,7km2, el cantón La Libertad con 25,2 km2 y el cantón Santa Elena tiene una superficie de 3.668,90 km2.

Su situación geográfica privilegiada permite, entre otras, fotografiar la mejor caída del sol, observar el salto de ballenas jorobadas desde tierra, encontrar 23 especies de aves playeras migratorias boreales en las piscinas de Ecuasal, practicar el surf en Montanita, una de las localidades más famosas del país; visitar la playa de Ballenita donde desembarcó Francisco Pizarro, conocer la única iglesia de más de cien años, construida en madera, visitar el primer pozo petrolero del país, probar un pan hecho con técnicas ancestrales, entrar en un lodo volcánico medicinal, esto es un ejemplo de todo lo que se puede hacer en la Península, en un solo día.

Sus límites comprenden, al Norte Manabí, al Este Guayas, al Sur Guayas, y al Oeste el Océano Pacifico.

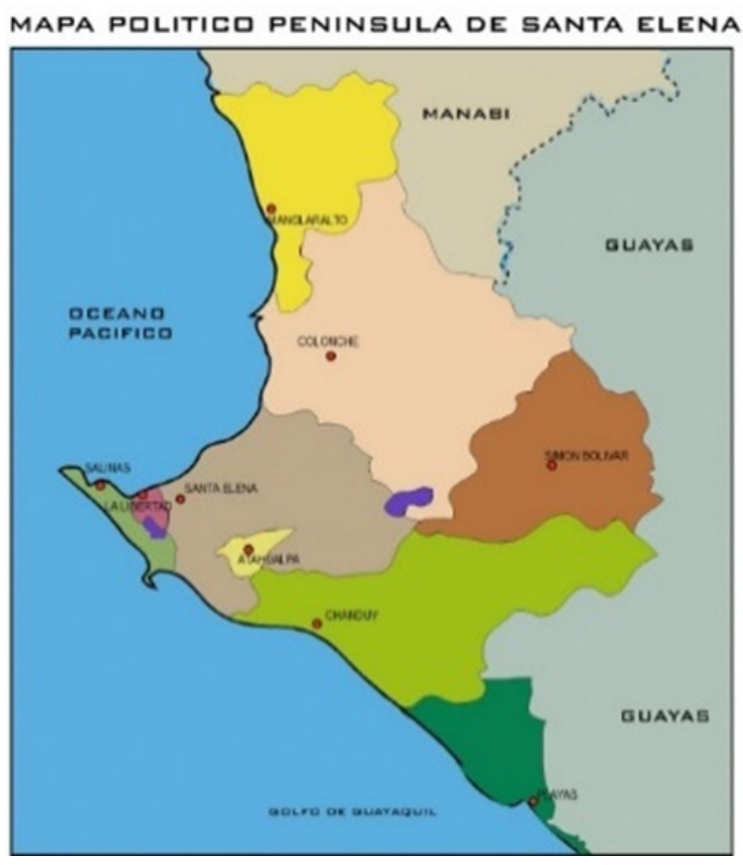

Imagen 1. Mapa de la provincia de Santa Elena

Los restos humanos más antiguos descubiertos 
en el Ecuador han sido encontrados en la Península de Santa Elena. Esta provincia estuvo poblada desde la prehistoria por la Cultura Las Vegas, que se asentó en los actuales territorios del Ecuador en el holoceno y pleistoceno tardío en la costa entre 8000 AC y 4600 AC. El nombre de Las Vegas obedece a un río que llena su cauce sólo cuando hay lluvias. Eran grupos seminómadas, organizados en bandas que deambulaban por las montañas en busca de recursos alimenticios.

Más tarde llegaría la Cultura Valdivia que se desarrolló entre el 3500 y el 1800AC en la costa del Ecuador. Esta cultura es la primera que demuestra conocimientos de alfarería, la agricultura del maíz, la textilería, la navegación y la práctica de ritos vinculados con la fertilidad de la tierra. Tenían la habilidad para trabajar la arcilla, entre las que se destacan las figuras antropomórficas llamadas hoy "Venus", que muestran un culto especial a la mujer, al hombre y a la fertilidad.

Luego se desarrolló la cultura Machalilla, quienes fueron ceramistas que fabricaron figuras femeninas, las más antiguas hechas en piedra y luego en barro, relacionadas con la fertilidad y la salud. El 18 de agosto de 1527, Pizarro y sus soldados desembarcaron en Ballenita, tierra que bautizó con este nombre debido a la gran cantidad de ballenas que encontró en sus aguas. Pizarro, recordando que el 18 de agosto era día de la emperatriz Santa Elena, nombró a esta tierra como "Santa Elena", que antes fue llamada "Sumpa", que quiere decir punta en el idioma Chimú .

La Península posee en general un clima cálido seco de $23^{\circ} \mathrm{C}$ a $25^{\circ} \mathrm{C}$. La temperatura más baja es de $15^{\circ} \mathrm{C}$ (julio y agosto). La temperatura máxima es de $39,5^{\circ} \mathrm{C}$ (febrero y marzo). Su economía principalmente en la pesquería artesanal, producción acuícola de camarón, agricultura de ciclo corto, extracción de petróleo, producción artesanal en madera y derivados, producción de sal marina y turismo.

Los platos típicos que se preparan en la Península mantienen una similitud en su preparación y en sus sabores, en todos los restaurantes, hoteles y huecas a lo largo de la Ruta del Spondylus. Entres sus platos típicos tenemos: ceviches (michulla, camarón, pulpo, pescado, concha, langosta, ostra, mixto), encebollado (pescado, mixto), viche de pescado, cazuela de mariscos, seco (chivo, pollo), entre otros.

En este contexto, contar con un programa cultural orientado a promover el trabajo voluntario internacional y el aprendizaje del idioma español en las comunidades de la Península de Santa Ele- na, en la convivencia con una familia nativa, con ese "cholo pescador" y a través de él, conocer sus costumbres, gastronomía, modo de vida, atractivos turísticos, etc., constituye un espacio propicio y estratégico que permite evidenciar que la interculturalidad es una dinámica de relación social, económica, ambiental, cultural y política entre los todos los actores de un territorio, pues el habitante peninsular también amplía su horizonte de conocimientos conviviendo con un habitante procedente de otros países.

Entre los beneficios y destrezas que los participantes adquieren luego de un voluntariado tenemos: aumento de la autoestima y confianza, desarrollo de nuevas habilidades, capacidad para mantener una conversación en ingles y/o español, cuenta con nueva visión del mundo y, contribuye a su madurez e independencia

El Programa contempla seis ejes; Turismo, Educación, Salud, Comunidad, Naturaleza y Gastronomía; cada uno de ellos está compuesto de varias opciones de voluntariado, el cual se esquematiza a continuación:

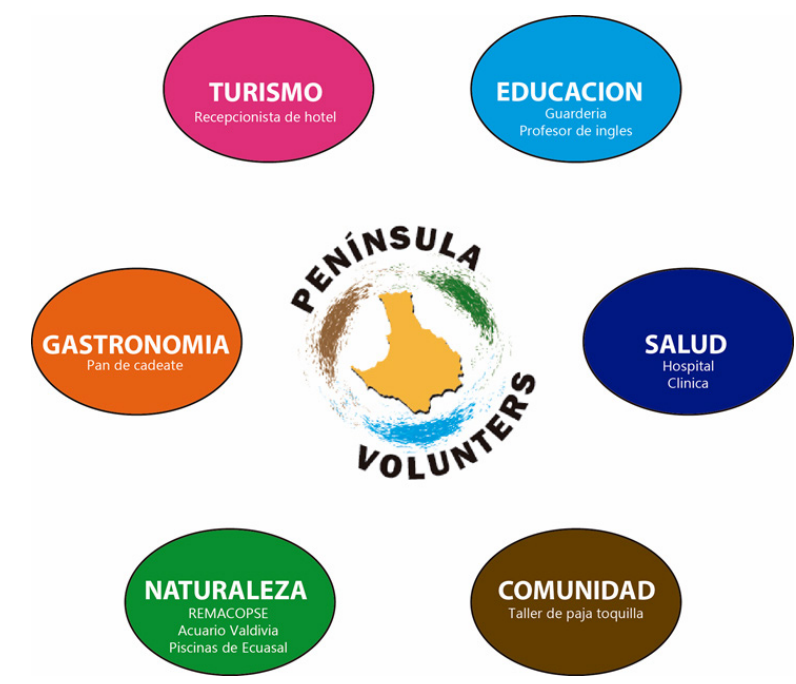
EDUCACION

Figura N. 2 Componentes del Plan

Educación: Guardería: Esta es una oportunidad donde podrá colaborar en actividades tales como: bailes, juegos, estimulación, y brindarles el estímulo que necesitan para el desarrollo de su autoestima y confianza.

Ubicación: Santa Elena

Profesor de inglés: El inglés es cada vez más necesario en todos los aspectos de la vida; sin embargo, uno de los problemas que tenemos los latinos es la pronunciación, debido a que el español no tiene la misma cantidad de vocales que el idioma inglés, y lo que hacemos es imitar los 
sonidos utilizando las vocales del español, lo que da como resultado una pronunciación deficiente. Como voluntariado de la enseñanza del idioma inglés, los alumnos tendrán una oportunidad para practicar fonética, corregir errores en su pronunciación y mantener una conversación.

\section{Ubicación: Salinas, La Libertad, Montañita}

Salud: Asistente en un hospital: Esta actividad tiene como objetivo facilitar el acercamiento del quehacer hospitalario; aprender a tratar a los pacientes con calidad y calidez, desarrollar habilidades como asistente de salud y practicar el español. Se realizarán tareas como brindar información a los visitantes, apoyar en trámite de ingreso, de alta, etc. Los departamentos donde se podrían incorporar sería: farmacia, fisioterapia, laboratorios, entre otros.

\section{Ubicación: La Libertad, Santa Elena}

Gastronomía: Panadero ancestral: Cocinar con leña ha sido una tradición en la Comuna Cadeate, más conocida como la "Capital del Pan", En este lugar se tendrá la oportunidad de aprender a elaborar y degustar un pan único hecho en horno de barro y leña. En el aroma y el sabor de este pan se encuentra la sabiduría ancestral de sus comuneros, quienes elaboran pan, roscas de sal, rosquitas, biscochos, suspiros, entre otros.

Ubicación: Muey, Cadeate.

Naturaleza: Parque Marino Valdivia: El Parque Marino, está compuesto por áreas y cada una consta de cinco grupos de acuarios y tanques: tanque Lobo Marino, tanque Pingüinos, tanque Tortugas Marinas, Tanque Tiburones y Rayas, Tanques de exhibición de peces pequeños, Aviario, un área de exhibición de cocodrilos. El acuario funciona desde hace unos 15 años. En la actualidad, la comuna maneja la instalación, con la ayuda de centros educativos superiores. Alí, además de proyectos de investigación y crianza de especies marinas, se rescata y se da tratamiento a animales decomisados en operativos de control o que han aparecido heridos. Entre sus pacientes están un lobo marino y un pingüino que ahora son exhibidos.

Proyecto Conservando Áreas Prioritarias para Aves Acuáticas, Piscinas Artificiales de Ecuasal: Son piscinas construidas por la empresa Ecuasal C.A. en los años 1950 para la producción de sal a nivel industrial, y es en estas piscinas donde se encuentran una gran cantidad de aves playeras migratorias y residentes. El gran número de aves que arriban a estas piscinas les ha merecido el reconocimiento internacional como Primer Refu- gio Ecuatoriano de Aves Playeras Migratorias de la Red Hemisférica de Reservas para Aves Playeras (RHRAP). La gestión de conservación de las piscinas se realiza en el marco del Proyecto Conservando Áreas Prioritarias para Aves Acuáticas de la Fundación Ecuatoriana Aves y Conservación / Bird Life en Ecuador. Este proyecto tiene tres líneas de trabajo estratégico: 1. Programa de monitoreo e investigación de poblaciones de aves acuáticas que se maneja desde 2004, hasta la actualidad, 2. Programa de Educación Ambiental y Comunicación y 3. Gestión Ambiental o de Restauración del Ecosistema.

Ecuasal es un sitio estratégico para el turismo científico y el aviturismo. Este tipo de voluntariado se ajusta, según Ana Agreda, Directora del Proyecto de Conservación en las Piscinas de Ecuasal, a los objetivos planteados en el Programa de Educación Ambiental y Comunicación del Plan de Conservación de Ecuasal, pues fomenta la formación de un grupo de voluntarios guardianes de la naturaleza que ayuden a identificar y mitigar problemas ambientales dentro de las áreas de influencia y promociona la formación de clubes de observación de aves

Comunidad: Taller de Secado de Paja Toquilla: Elaborar los famosos sombreros de paja toquilla a mano puede tardar de 3 a 6 meses y muchas veces se lo conoce en el exterior como "Panama hat", pero lo curioso es que son hechos en Ecuador y su materia prima es cultivada en la península de Santa Elena. Al escoger esta opción, conocerás el sitio donde se cultiva la materia prima, el proceso de su cultivo, el desechado, la limpieza, cocción, eliminación del color verde, la sahumada, el secado, su clasificación, entre otros.

Ubicación: Barcelona, Sinchal, Dos Mangas (Ruta del Spondylus).

Turismo: Recepcionista de hotel: Si desea es adquirir experiencia en el campo de la hotelería, esta es una excelente oportunidad para trabajar como recepcionista en un lujoso hotel de la Península, donde podrá adquirir habilidades y conocimientos referentes a la industria de la hotelería.

Ubicación: Salinas, La Libertad, Montañita.

\section{Conclusiones}

La Península de Santa Elena, cuenta con asentamientos poblacionales en sus parroquias que son referentes históricos, culturales y turísticos, que conviven en un modelo de desarrollo urbano propio, donde se puede encontrar una cultura con fuertes rasgos tradicionales, provenientes de sus poblaciones ancestrales, así como de migrantes de todo el país y de la influencia cultural de 
personas que han llegado de otros países; este conjunto de personas que habitan en la Península de Santa Elena, conjuntamente con su clima y atractivos turísticos, la definen como una población propicia para un Programa de Voluntariado Internacional que potencie la interculturalidad.

La interculturalidad, entendida como un proceso social, plantea la necesidad de conocerla y reconocerla como válida y productora de aportes a las culturas, lo que permite aceptarnos con nuestras diferencias, apreciar nuestras diversas formas de ser y ver la vida y, construir un mundo más humano. Esto implica: cambios personales y de actitud de la sociedad, en su conjunto. Asumir plenamente la interculturalidad, implica confiar en que es posible construir relaciones más armónicas, respetando la diversidad como una práctica básica cotidiana

Esta propuesta de Programa de Voluntariado Internacional, propende una entrada de reflexión para entender la complejidad de la interculturalidad, a través de la convivencia cotidiana de diferentes manifestaciones culturales de la Península de Santa Elena. Por otro lado, procura la reactivación del capital social, cultural y productivo que incentive el fortalecimiento de la identidad del habitante peninsular a nivel barrial y parroquial, posibilitando la construcción de sociedades incluyentes.

\section{Referencias Bibliográficas}

ADORNO, Rolena. "El sujeto de Crítica Literaria colonial y la construcción cultural de la alteridad”. En: Revista Latinoamericana, Año 14, No. 28, Historia, Sujeto Social y Discurso Poético en la Colonia. (1998).

AGREDA, Ana. Plan de Conservación de las Piscinas Artificiales de Ecuasal periodo 2012 - 2015 y Estudio de Capacidad de Carga Turística. Aves y Conservación - BirdLife en Ecuador y Ecuatoriana de Sal y Productos Químicos C.A. Guayaquil, Ecuador. Pp. 108. (2012)

BOAVENTURA, De Santos; "Cuando los excluidos tienen derecho: justicia pluriculturalidad e interculturalidad en Bolivia y Ecuador" Ediciones Abya-Yala y Fundación Rosa Luxemburgo, La Paz. (2012)

GUERRERO, Andrés; Compilador, Etnicidades; FLACSO-ILDIS. Quito-Ecuador. (2000).

HAASE, Ben; "Aves marinas del Ecuador Continental y acuáticas de las piscinas artificiales de Ecuasal”, Graficas Hernández, Guayaquil-Ecua- dor.

MORIN, Edgar; "La vía para el futuro de la humanidad”; PAIDÓS, Barcelona-España. (2011)

PRIETO, Mercedes; editora. "Mujeres ecuatorianas y las oportunidades1990-2004"CONAMU, FLACSO, UNIFEM. (2005).

TUAZA, Luis; "Comunidades indígenas de la provincia de Chimborazo, Ecuador: permanencia de la sombra del régimen de hacienda".(2014)

WAISMANTEL, Mary; "Alimentación, género y pobreza en los Andes ecuatorianos" Abya-Yala. Quito-Ecuador. (1994).

WALSH, Catherine; "Interculturalidad crítica y pedagogía de-colonial: apuestas (des)de el in-surgir, re-existir y re-vivir"(2009).

\section{Agradecimiento}

A Ana Agreda, Directora del proyecto de las Piscinas Artificiales de Ecuasal, a Félix Lavayen, gestor cultural de la Península, a Douglas Dillon, creador de la Ruta del Sol y a Mercedes Guerra, jefa del departamento de turismo de la Prefectura de Santa Elena. 